\title{
Teleportation of the Relativistic Quantum Field
}

\author{
R.Laiho ${ }^{\dagger}$, S.N.Molotkov ${ }^{\ddagger}$, S.S.Nazin ${ }^{\ddagger}$ \\ ${ }^{\dagger}$ Wihuri Physical Laboratory, \\ Department of Physics, University of Turku, 20014 Turku, Finland \\ ${ }^{\ddagger}$ Institute of Solid State Physics of Russian Academy of Sciences, \\ Chernogolovka, Moscow District, 142432 Russia
}

\begin{abstract}
The process of teleportation of a completely unknown one-particle state of a free relativistic quantum field is considered. In contrast to the non-relativistic quantum mechanics, the teleportation of an unknown state of the quantum field cannot be in principle described in terms of a measurement in a tensor product of two Hilbert spaces to which the unknown state and the state of the EPR-pair belong. The reason is of the existence of a cyclic (vacuum) state common to both the unknown state and the EPR-pair. Due to the common vacuum vector and the microcausality principle (commutation relations for the field operators), the teleportation amplitude contains inevitably contributions which are irrelevant to the teleportation process. Hence in the relativistic theory the teleportation in the sense it is understood in the non-relativistic quantum mechanics proves to be impossible because of the impossibility of the realization of the appropriate measurement as a tensor product of the measurements related to the individual subsystems so that one can only speak of the amplitude of the propagation of the field as a whole.
\end{abstract}

PACS numbers: 03.67.-a, 03.65.Bz, 42.50Dv

One of the fundamental results of the non-relativistic quantum information theory consists in the possibility of the teleportation of an unknown quantum state by means of a quantum communication channel realized by a non-local entangled state (an EPR-pair [1]) used together with a classical communication channel [2].

When the unknown quantum state $|\psi\rangle_{s}$ belongs to a finite-dimensional Hilbert space $\left(\operatorname{dim} \mathcal{H}_{s}<\right.$ $\infty)$, the teleportation can be preformed ideally and with the unit probability employing an EPRpair with finite energy. On the other hand, if the state space of the teleported system is infinitedimensional, $\left(\operatorname{dim} \mathcal{H}_{s}=\infty\right)$, the ideal teleportation formally requires an EPR-pair with infinite energy $[3,4]$. However, the teleported state can be made arbitrarily close to the input unknown state with the probability arbitrarily close to unit by increasing the energy of the EPR-pair and thus making the EPR correlations more and more close the ideal ones.

The non-relativistic quantum mechanics yields only an approximate description of the reality. A more correct and complete description is provided by quantum field theory (since the relativistic quantum mechanics does not allow any sensible physical interpretation, the relativistic theory arises from the very beginning as a quantum field theory). Therefore, it is interesting to consider the possibility of teleportation of a completely unknown state of the relativistic quantum field. In addition, although all the teleportation experiments carried out so far are performed with photons which are essentially relativistic particles, they are always interpreted within the framework of the non-relativistic quantum mechanics.

In the rest of the paper, considering a simple example, we shall show that in the relativistic quantum field theory the teleportation of even a one-particle state of free quantum field cannot be achieved in the sense it is understood in the non-relativistic quantum mechanics. The latter actually follows from the existence of a vacuum (cyclic) state which is common to both the completely unknown state to be teleported and the EPR pair together with the microcausality principle (commutation or anticommutation relations for the field operators).

For convenience we shall briefly remind the teleportation procedure in the non-relativistic case.

Suppose that we are given an unknown quantum state $|\psi\rangle_{s} \in \mathcal{H}_{s}\left(\operatorname{dim} \mathcal{H}_{s}<\infty\right)$ and a maximally entangled EPR-pair $|\psi\rangle_{E P R} \in \mathcal{H}_{12}=\mathcal{H}_{1} \otimes \mathcal{H}_{2}\left(\operatorname{dim} \mathcal{H}_{12}<\infty\right)$. The EPR-pair is a composite system consisting of two particles with the state spaces $\mathcal{H}_{1}$ and $\mathcal{H}_{2}$. To achieve the teleportation, one performs a joint measurement on the unknown quantum state and one of the particles of the EPR-pair described 
by an identity resolution in $\mathcal{H}=\mathcal{H}_{s} \otimes \mathcal{H}_{12}$ over some measurable outcome space $\Theta$ (which is discrete, $\Theta=\sum_{i} \theta_{i}$, for the teleportation of the states belonging to finite-dimensional spaces). The measurement is defined by the identity resolution

$$
I_{s 12}=\sum_{i} \mathcal{M}\left(\theta_{i}\right)=\sum_{i} I_{1} \otimes \mathcal{M}_{2 s}\left(\theta_{i}\right)
$$

If the measurement yields the $i$-th outcome, the subsystem 1 is found in a new state

$$
\rho_{1}^{i}=\frac{\operatorname{Tr}_{2 s}\left\{\mathcal{M}\left(\theta_{i}\right)\left(\rho_{s} \otimes \rho_{E P R}\right)\right\}}{\operatorname{Tr}_{12 s}\left\{\mathcal{M}\left(\theta_{i}\right)\left(\rho_{s} \otimes \rho_{E P R}\right)\right\}}
$$

To within a unitary rotation $U_{i}$ which only depends on the measurement outcome $i$ and does not depend on the unknown state, the state (2) coincides with the unknown input state:

$$
\tilde{\rho}_{1}=\rho_{s} \quad \tilde{\rho}_{1}=U_{i} \rho_{1}^{i} U_{i}^{-1}
$$

The non-relativistic teleportation procedure substantially employs the fact that the Hilbert state space of each subsystem can be accessed separately.

In the non-relativistic quantum mechanics physically different systems are treated in the same way in the sense that any two systems are formally considered to be identical if their state spaces are identical (isomorphic). Therefore, formally, teleported is the unknown state vector rather than the particle itself. There are no rules that prohibit the superposition of the states belonging to physically different subsystems. In the quantum field theory the situation is completely different.

Let us now turn to the teleportation of an unknown state of a free quantum field. For simplicity we shall first consider the teleportation of a one-particle state of the free scalar quantum field, although the most interesting is perhaps the case of the gauge (photon) field. To avoid unnecessary technical details associated with the indefinite metrics, we shall restrict our analysis to the scalar field. All the remarks concerning the scalar field teleportation are also relevant to photon teleportation [9] (we mean the teleportation of a completely unknown one-photon state when not only the polarization state but also the wave packet shape is unknown).

The states of a relativistic quantum system are described by the rays in the physical Hilbert space $\mathcal{H}$ where a unitary representation of the covering Poincaré group is realized $[5,6]$. The local quantum field $\varphi(\hat{x})$ (here $\hat{x}=(t, \mathbf{x})$ is a point in the Minkowski space-time) is defined as a tensor (if the field has more than one component) operator-valued distribution. To be more precise, corresponding to any function (or a set of functions, if the field is multicomponent) $f(\hat{x}) \in \mathcal{J}(\hat{x})$, where $\mathcal{J}(\hat{x})$ is the space of test infinitely differentiable functions decreasing together with all their derivatives at the infinity faster than any polynomial $[5,6])$, is the operator symbolically written as

$$
\varphi(f)=\sum_{j=1}^{r} \varphi_{j}\left(f_{j}\right)=\sum_{j=1}^{r} \int \varphi_{j}(\hat{x}) f_{j}(\hat{x}) d \hat{x} .
$$

The operators $\varphi(f)$ and $\varphi^{*}(f)$ have a common domain which does not depend on $f(\hat{x})$, is dense in $\mathcal{H}$, and is invariant under the action of the field operators, $\varphi(f) \Omega \subset \Omega\left(\varphi^{*}(f) \Omega \subset \Omega\right)$. For any vectors $|\phi\rangle,|\psi\rangle \in \Omega \subset \mathcal{H}$ the quantity $\langle\phi|\varphi(f)| \psi\rangle$ is a distribution from $\mathcal{J}^{*}(\hat{x})\left(\mathcal{J}^{*}(\hat{x})\right.$ is the space of distributions conjugate to $\mathcal{J}(\hat{x}))$.

The space $\Omega$ contains a cyclic vector, called the vacuum state, $|0\rangle \in \Omega$ such that the set of all polynomials $\mathcal{P}(\varphi, f)$ constitute an operator algebra with involution whose action on $|0\rangle \in \Omega$ generates the entire space $\Omega$. The field operator algebra is defined as

$$
\begin{gathered}
\mathcal{P}(\varphi, f)=f_{0}+\sum_{n=1}^{\infty} \iint \ldots \int \varphi\left(\hat{x}_{1}\right) \varphi\left(\hat{x}_{2}\right) \ldots \varphi\left(\hat{x}_{n}\right) f\left(\hat{x}_{1}, \hat{x}_{2}, \ldots, \hat{x}_{n}\right) d \hat{x}_{1} d \hat{x}_{2} \ldots d \hat{x}_{n} \\
f\left(\hat{x}_{1}, \hat{x}_{2}, \ldots, \hat{x}_{n}\right) \in \mathcal{J}\left(\hat{x}^{n}\right) .
\end{gathered}
$$


The fact that the field operators form an algebra implies that any observable can be expressed through the field operators $[5,6]$.

The unsmeared field operators $\varphi(\hat{x})$ map the regular states from $\Omega$ to the generalized states $\mathcal{P}(\varphi(\hat{x})) \Omega \subset \Omega^{*}\left(\Omega^{*}\right.$ is the conjugate space to $\Omega$ consisting of all the linear functionals defined on $\Omega$ and continuous with respect to the scalar product in $\mathcal{H}$ ). The microcausality principle is also postulated; to be more precise, the field operators are assumed to commute (anticommute) if the supports of their corresponding functions $f(\hat{x}), g(\hat{y})$ are separated by a space-like interval $\left(\operatorname{supp} f(\hat{x}) \cdot g(\hat{y}) \in(\hat{x}-\hat{y})^{2}<0\right)$, i.e. for any vector $|\psi\rangle \in \Omega$ we have

$$
[\varphi(f), \varphi(g)]_{ \pm}|\psi\rangle=0, \quad(\hat{x}-\hat{y})^{2}<0 .
$$

The expression (6) is interpreted as the impossibility of any causal relation between the measurements performed in the domains separated by a space-like interval since no interaction can propagate faster than light.

Further, the requirements that the system states are described by the rays in the Hilbert where a Poincaré group representation is realized and the spectrum of the group generators in the momentum representation lies in the front part of the light cone imply that the Lorentz-covariant quantum field can only be realized as an operator valued distribution rather than the field of operators $\varphi(\hat{x})$ acting in $\mathcal{H}[5,6]$.

The interpretation of a quantum field as a field of operators acting in $\mathcal{H}$ is only consistent with the trivial two-point function $\left\langle 0\left|\varphi^{-}(\hat{x}) \varphi^{+}(\hat{y})\right| 0\right\rangle=$ const and results in an obvious violation of the microcausality principle. The smearing function $f(\hat{x})$ can be interpreted (with some reservations) as the amplitude ("shape") of the one-particle packet.

Let us now construct the EPR-state, the one-particle state of the scalar to be teleported, and the corresponding measurement for the relativistic case emphasizing the differences from the nonrelativistic theory.

The state of the EPR-pair is described by the vector $|\psi\rangle_{E P R} \in \Omega \in \mathcal{H}$ in the subspace of the two-particle states. The most general form of the relevant vectors is

$$
|\psi\rangle_{E P R}=\mathcal{P}_{2}(\varphi, \mathcal{F})|0\rangle=\iint d \hat{x}_{1} d \hat{x}_{2} \mathcal{F}\left(\hat{x}_{1}, \hat{x}_{2}\right) \varphi^{+}\left(\hat{x}_{1}\right) \varphi^{+}\left(\hat{x}_{2}\right)|0\rangle
$$

where

$$
\begin{gathered}
\varphi^{ \pm}(\hat{x})=\frac{1}{(2 \pi)^{3 / 2}} \int \mathrm{e}^{\mp i \hat{k} \hat{x}} \theta\left(k^{0}\right) \delta\left(\hat{k}^{2}-m^{2}\right) a^{ \pm}(\hat{k}) d \hat{k}=\frac{1}{(2 \pi)^{3 / 2}} \int_{V_{m}^{+}} \mathrm{e}^{\mp i \hat{k} \hat{x}} a^{ \pm}(\mathbf{k}) \frac{d \mathbf{k}}{\sqrt{2 k^{0}}} \\
\hat{k} \hat{x}=k^{0} x^{0}-\mathbf{k} \cdot \mathbf{x}, \quad k^{0}=\sqrt{\mathbf{k}^{2}+m^{2}} .
\end{gathered}
$$

The symbol $V_{m}^{+}$in the second integral is kept to emphasize the fact that contributing to the integral are only the values at the mass shell inside the front part of the light cone $k^{0}>0$. The ideal EPR correlations correspond to the case where

$$
\tilde{\mathcal{F}}\left(\hat{x}_{1}, \hat{x}_{2}\right)=\delta\left(x_{1}^{0}-x^{0}\right) \delta\left(x_{2}^{0}-x^{0}\right) \delta\left(\mathbf{x}_{1}-\mathbf{x}_{2}\right) \operatorname{const}\left(\mathbf{x}_{1}+\mathbf{x}_{2}\right) .
$$

However, the function (8) does not belong to the space of test functions $\mathcal{J}\left(\hat{x}^{2}\right)$ and should be understood as a limit of functions $\mathcal{F}\left(\hat{x}_{1}, \hat{x}_{2}\right) \in \mathcal{J}\left(\hat{x}^{2}\right), \mathcal{F}\left(\hat{x}_{1}, \hat{x}_{2}\right) \rightarrow \tilde{\mathcal{F}}\left(\hat{x}_{1}, \hat{x}_{2}\right)$. The ideal EPR pair correspond to the generalized state vector $|\psi\rangle_{E P R} \in \Omega^{*}$ of the form

$$
|\psi\rangle_{E P R}=\frac{1}{(2 \pi)^{3}} \int_{V_{m}^{+}} \frac{d \mathbf{k}}{2 k^{0}} \mathrm{e}^{-2 i k^{0} x^{0}} a^{+}(\mathbf{k}) a^{+}(-\mathbf{k})|0\rangle .
$$

This state is an analogue of the ideal EPR state [1] in the non-relativistic case for the composite system consisting of two particles 1 and 2,

$$
|\psi\rangle_{E P R}=\frac{1}{(2 \pi)^{3}} \int d \mathbf{k}|\mathbf{k}\rangle_{1} \otimes|-\mathbf{k}\rangle_{2}
$$


where $|\mathbf{k}\rangle_{1,2}$ are the generalized eigenvectors of the momentum operator, and $|\mathbf{k}\rangle_{1,2} \in \mathcal{J}^{*}(\mathbf{k})\left(\mathcal{J}^{*}(\mathbf{k})\right.$ is the distribution space conjugate to $\mathcal{J}(\mathbf{k}))$. Accordingly, in the position representation the state is written as

$$
|\psi\rangle_{E P R}=\frac{1}{(2 \pi)^{3}} \int d \mathbf{x}|\mathbf{x}\rangle_{1} \otimes|\mathbf{x}\rangle_{2}
$$

where $|\mathbf{x}\rangle_{1,2}$ are the generalized eigenvectors of the position operator, and $|\mathbf{x}\rangle_{1,2} \in \mathcal{J}^{*}(\mathbf{x})\left(\mathcal{J}^{*}(\mathbf{x})\right.$. The Fourier transform is known to map the space of distributions $\mathcal{J}^{*}(\mathbf{k})$ onto $\mathcal{J}^{*}(\mathbf{x})$.

Qualitatively, at the intuitive level, the state (9) with $\tilde{\mathcal{F}}$ from (8) can be interpreted as the creation of two particles with $\mathbf{x}_{1}=\mathbf{x}_{2}$ at time $x^{0}$ from vacuum, simultaneously at the entire space (because of the presence of a factor const $\left.\left(\mathbf{x}_{1}+\mathbf{x}_{2}\right)\right)$. The EPR state is essentially non-local.

The one-particle packet state of the quantum field can be written as

$$
|\psi\rangle_{s}=\varphi^{+}(f)|0\rangle=\int d \hat{x} f(\hat{x}) \varphi^{+}(\hat{x})|0\rangle=\frac{1}{(2 \pi)^{3 / 2}} \int_{V_{m}^{+}} \frac{d \mathbf{k}}{2 k^{0}} f(\mathbf{k}) a^{+}(\mathbf{k})|0\rangle,
$$

where $f(\mathbf{k})$ is the packet amplitude in the $\mathbf{k}$-representation. The state is defined by the equivalence class to which the function $f(\hat{x})$ belongs. Different functions $f(\hat{x})$ which have the same values on the mass shell define the same states.

The non-relativistic analogue of the packet is the state

$$
|\psi\rangle_{s}=\int d \mathbf{k} f(\mathbf{k})|\mathbf{k}\rangle_{s}
$$

belonging in the non-relativistic case to the Hilbert state space of the particle to be teleported, $|\psi\rangle_{s} \in \mathcal{H}_{s}$.

Because of the existence of a common vacuum state in the quantum field theory, the vector corresponding to the system "EPR pair + teleported state" should be written as

$$
|\Psi\rangle=\varphi^{+}(f) \mathcal{P}\left(\varphi^{+}, \mathcal{F}\right)|0\rangle=\iiint d \hat{x}_{1} d \hat{x}_{2} d \hat{x} \mathcal{F}\left(\hat{x}_{1}, \hat{x}_{2}\right) f(\hat{x}) \varphi^{+}\left(\hat{x}_{1}\right) \varphi^{+}\left(\hat{x}_{2}\right) \varphi^{+}(\hat{x})|0\rangle,
$$

The existence of a common vacuum state in the relativistic quantum field theory results in a fundamental difference between the relativistic and non-relativistic cases. In contrast to the nonrelativistic case where $|\psi\rangle_{s} \otimes|\psi\rangle_{E P R} \in \mathcal{H}_{s} \otimes \mathcal{H}_{12}=\mathcal{H}_{s} \otimes \mathcal{H}_{1} \otimes \mathcal{H}_{2}$, the three-particle states of the quantum field $|\Psi\rangle \in \Omega \subset \mathcal{H}$. (Of course, a different representation of the state space $\mathcal{H}=\oplus_{n} \operatorname{Sym} \otimes^{n} \mathcal{H}_{1}$ as a direct sum of the symmetrized tensor products of the one-particle Fock spaces introduces no changes because of the existence of a common cyclic vacuum vector.)

In addition, in the quantum field theory the states are all essentially non-localizable in the sense that, as it was already established long ago (see e.g. Ref.[7]), it is impossible to construct a state with a compact support in the $\mathbf{x}$-representation using the normalized functions $f(\mathbf{k})$ defined on the mass shell (although the states with the fall off arbitrarily close to the exponential one at the infinity can be constructed) [8]. In some cases one can perhaps approximately assume that the states of a composite system localized to within the exponential tails in distant spatial domains can be regarded as the states defined in the tensor product of the corresponding state spaces which formally have different vacuum states. However, this assumption is certainly wrong if the composite systems in entangled states and their measurements are to be considered. This is exactly the case in the teleportation problem. Moreover, if the state space of a composite system is described as a tensor product of the constituent system state spaces, the microcausality principle (commutation relations) is inevitably violated since the operators acting in different Hilbert spaces (factors in the tensor product) are certainly always commuting independently of their position in the Minkowski space; to be more precise, the operators even do not "know" about each other.

Let us now construct the appropriate measurement. Since in the relativistic case the states are also described by the rays in the Hilbert space (just as in the non-relativistic quantum mechanics), the measurements are also described by the positive operator valued identity resolutions. 
In the non-relativistic case the measurement used in the teleportation procedure is described by an identity resolution in $\mathcal{H}=\mathcal{H}_{s} \otimes \mathcal{H}_{1} \otimes \mathcal{H}_{2}$. Let $\Theta$ be a measurable space of possible outcomes with the measure $d \theta$; then

$$
I_{s 12}=I_{s} \otimes I_{1} \otimes I_{2}=\int_{\Theta} \mathcal{M}_{\mathcal{H}}(d \theta)=I_{1} \otimes \int_{\Theta} \mathcal{M}_{2 s}(d \theta)=I_{1} \otimes I_{2 s}
$$

The measurement (15) is only performed on one of the particles in the EPR-pair and the particle in the unknown state to be teleported while the second particle in the EPR-pair (the factor $I_{1}$ ) is not involved in the measurement itself. It is important for the teleportation procedure that the identity resolution in the entire state space of the three subsystems can be expressed as a tensor product of the corresponding identity resolutions in $\mathcal{H}_{1}$ and $\mathcal{H}_{s 2}=\mathcal{H}_{s} \otimes \mathcal{H}_{2}$, which implicitly assumes the access to the individual subsystems.

For the relativistic quantum field, the identity resolution in the three-particle states subspace cannot be in any way represented as a tensor product of the appropriate identity resolutions in the one-particle and two-particle subspaces. Such a measurement should only be constructed as a general identity resolution in the entire three-particle states subspace.

It is first instructive to examine the measurement used in the teleportation of a one-particle packet in the non-relativistic case:

$$
\mathcal{M}_{\mathcal{H}}(d \theta)=I_{1} \otimes \mathcal{M}_{2 s}(d \theta)=I_{1} \otimes\left|\Phi_{\mathbf{X P}}\right\rangle_{2 s}{ }_{2 s}\left\langle\Phi_{\mathbf{X P}}\right| \frac{d \mathbf{X} d \mathbf{P}}{(2 \pi)^{3}}
$$

where the space of possible outcomes is the set $\Theta=\left\{\mathbf{X} \times \mathbf{P} \in \mathbf{R}_{\mathbf{X}} \times \mathbf{R}_{\mathbf{P}}\right\}$.

Here $\mathbf{X}$ is the sum of the particle positions $\mathbf{X}=\mathbf{x}_{2}+\mathbf{x}_{s}, \mathbf{P}=\mathbf{p}_{2}-\mathbf{p}_{s}$ is the difference of their momenta, and

$$
\left|\Phi_{\mathbf{X P}}\right\rangle_{2 s}=\int d \mathbf{k} \mathrm{e}^{i \mathbf{k X}}|\mathbf{k}\rangle_{2} \otimes|\mathbf{k}+\mathbf{P}\rangle_{s}
$$

It is easy to check that $\mathcal{M}(d \mathbf{X} d \mathbf{P})$ is actually an identity resolution in $\mathcal{H}_{2} \otimes \mathcal{H}_{s}$; indeed,

$$
\begin{gathered}
I_{2 s}=I_{2} \otimes I_{s}=\int\left|\Phi_{\mathbf{X P}}\right\rangle_{2 s}{ }_{2 s}\left\langle\Phi_{\mathbf{X P}}\right| \frac{d \mathbf{X} d \mathbf{P}}{(2 \pi)^{3}}= \\
\left.\iint d \mathbf{k}_{1} d \mathbf{k}_{2}\left(\left|\mathbf{k}_{1}\right\rangle_{2} \otimes\left|\mathbf{k}_{2}\right\rangle_{s}\right){ }_{(s}\left\langle\mathbf{k}_{2}\right| \otimes{ }_{2}\left\langle\mathbf{k}_{1}\right|\right) .
\end{gathered}
$$

A similar identity resolution for the relativistic quantum field in the subspace of two-particle states is

$$
I=\int_{V_{m}^{+}} \int_{V_{m}^{+}} \frac{d \mathbf{k}_{1}}{2 k_{1}^{0}} \frac{d \mathbf{k}_{2}}{2 k_{2}^{0}}\left(a^{+}\left(\mathbf{k}_{1}\right) a^{+}\left(\mathbf{k}_{2}\right)|0\rangle\right)\left(\langle 0| a^{-}\left(\mathbf{k}_{2}\right) a^{-}\left(\mathbf{k}_{1}\right)\right) .
$$

Let us first write down the analogue of the measurement (17) and then complete it to the identity resolution in the subspace of three-particle states. The corresponding measurement can be represented in the form

$$
\begin{gathered}
\mathcal{M}(d \theta)= \\
\left(\iint d \hat{\xi}_{1} d \hat{\xi}_{2} \Phi\left(\theta, \hat{\xi}_{1}, \hat{\xi}_{2}\right) \varphi^{+}\left(\hat{\xi}_{1}\right) \varphi^{+}\left(\hat{\xi}_{2}\right)|0\rangle\right)\left(\iint d \hat{\xi}_{1}^{\prime} d \hat{\xi}_{2}^{\prime} \Phi^{*}\left(\theta, \hat{\xi}_{1}^{\prime}, \hat{\xi}_{2}^{\prime}\right)\langle 0| \varphi^{-}\left(\hat{\xi}_{1}^{\prime}\right) \varphi^{-}\left(\hat{\xi}_{2}^{\prime}\right)\right) d \theta
\end{gathered}
$$

which should give the identity resolution (19), i.e.

$$
\begin{gathered}
I=\int \mathcal{M}(d \theta)=\left(\iint d \hat{\xi}_{1} d \hat{\xi}_{2} \varphi^{+}\left(\hat{\xi}_{1}\right) \varphi^{+}\left(\hat{\xi}_{2}\right)|0\rangle\right)\left(\iint d \hat{\xi}_{1}^{\prime} d \hat{\xi}_{2}^{\prime}\langle 0| \varphi^{-}\left(\hat{\xi}_{1}^{\prime}\right) \varphi^{-}\left(\hat{\xi}_{2}^{\prime}\right)\right) \\
\left(\int d \theta \Phi\left(\theta, \hat{\xi}_{1}, \hat{\xi}_{2}\right) \Phi^{*}\left(\theta, \hat{\xi}_{1}^{\prime}, \hat{\xi}_{2}^{\prime}\right)\right)
\end{gathered}
$$

which implies the conditions

$$
\Phi\left(\theta, \hat{\xi}_{1}, \hat{\xi}_{2}\right)=\delta\left(\xi_{1}^{0}-\xi^{0}\right) \delta\left(\xi_{2}^{0}-\xi^{0}\right) \Phi\left(\theta, \boldsymbol{\xi}_{1}, \boldsymbol{\xi}_{2}\right)
$$




$$
\int d \theta \Phi\left(\theta, \boldsymbol{\xi}_{1}, \boldsymbol{\xi}_{2}\right), \Phi^{*}\left(\theta, \boldsymbol{\xi}_{1}^{\prime}, \boldsymbol{\xi}_{2}^{\prime}\right)=\delta\left(\boldsymbol{\xi}_{1}-\boldsymbol{\xi}_{2}\right) \delta\left(\boldsymbol{\xi}_{1}^{\prime}-\boldsymbol{\xi}_{2}^{\prime}\right)
$$

It should be emphasized that the time $\xi^{0}$ is the same for $\hat{\xi}_{1}, \hat{\xi}_{2}$ and $\hat{\xi}_{1}^{\prime}, \hat{\xi}_{2}^{\prime}$. We shall not dwell on the interpretation of the measurement (20) and not only that this measurement can be considered as a non-local measurement in the position representation performed at time $\xi^{0}$.

The conditions (19-22) are satisfied if $\Phi$ is chosen in the from

$$
\Phi\left(\theta, \boldsymbol{\xi}_{1}, \boldsymbol{\xi}_{2}\right)=\delta\left(\boldsymbol{\xi}_{1}-\boldsymbol{\xi}_{2}\right) \mathrm{e}^{i \mathbf{P} \boldsymbol{\xi}_{1}}, \quad \theta=(\mathbf{X}, \mathbf{P}),
$$

where $\mathbf{X}, \mathbf{P}$ have the same meaning as in the non-relativistic case.

Finally, one obtains

$$
\begin{gathered}
\mathcal{M}(d \mathbf{X} d \mathbf{P})= \\
\left(\int_{V_{m}^{+}} \frac{d \mathbf{k}}{\sqrt{2 k^{0}(\mathbf{k})} \sqrt{2 k^{0}(\mathbf{k}+\mathbf{P})}} \mathrm{e}^{i \mathbf{k P}-i\left(k^{0}(\mathbf{k})+k^{0}(\mathbf{k}+\mathbf{P})\right) \xi^{0}} a^{+}(\mathbf{k}) a^{+}(\mathbf{k}+\mathbf{P})|0\rangle\right) \\
\left(\int_{V_{m}^{+}} \frac{d \mathbf{k}^{\prime}}{\sqrt{2 k^{0}\left(\mathbf{k}^{\prime}\right)} \sqrt{2 k^{0}\left(\mathbf{k}^{\prime}+\mathbf{P}\right)}} \mathrm{e}^{i \mathbf{k}^{\prime} \mathbf{P}+i\left(k^{0}\left(\mathbf{k}^{\prime}\right)+k^{0}\left(\mathbf{k}^{\prime}+\mathbf{P}\right)\right) \xi^{0}}\langle 0| a^{-}\left(\mathbf{k}^{\prime}+\mathbf{P}\right) \mathbf{a}^{-}\left(\mathbf{k}^{\prime}\right)\right) \frac{d \mathbf{X} d \mathbf{P}}{(2 \pi)^{3}} .
\end{gathered}
$$

We shall further need the following identity resolution in the subspace of one-particle states of the quantum field:

$$
I_{1}=\int_{V_{m}^{+}} \frac{d \mathbf{k}}{2 k^{0}}\left(a^{+}(\mathbf{k})|0\rangle\right)\left(\langle 0| a^{-}(\mathbf{k})\right)=\int d \mathbf{x}\left(\varphi^{+}(\hat{x})|0\rangle\right)\left(\langle 0| \varphi^{-}(\hat{x})\right) .
$$

The complete measurement in the subspace of the states of the composite system consisting of the EPR-pair and the packet to be teleported is

$$
\begin{aligned}
& \mathcal{M}_{\mathcal{H}}(d \theta)= \\
& \int d \mathbf{x}\left(\iint d \boldsymbol{\xi}_{1} d \boldsymbol{\xi}_{2} \Phi\left(\theta, \boldsymbol{\xi}_{1}, \boldsymbol{\xi}_{2}\right) \varphi^{+}\left(\hat{\xi}_{1}\right) \varphi^{+}\left(\hat{\xi}_{2}\right) \varphi^{+}(\hat{x})|0\rangle\right) \\
& \left(\iint d \boldsymbol{\xi}_{1}^{\prime} d \boldsymbol{\xi}_{2}^{\prime} \Phi^{*}\left(\theta, \boldsymbol{\xi}_{1}^{\prime}, \boldsymbol{\xi}_{2}^{\prime}\right)\langle 0| \varphi^{-}(\hat{x}) \varphi^{-}\left(\hat{\xi}_{1}^{\prime}\right) \varphi^{-}\left(\hat{\xi}_{2}^{\prime}\right)\right) d \theta=
\end{aligned}
$$

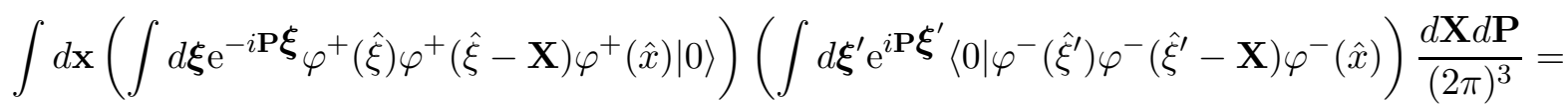

$$
\begin{aligned}
& \int d \mathbf{x}\left(\varphi^{+}(\hat{x})\left|\Phi_{\mathbf{X P}}\right\rangle\right)\left(\left\langle\Phi_{\mathbf{X P}}\right| \varphi^{-}(\hat{x})\right) \frac{d \mathbf{X} d \mathbf{P}}{(2 \pi)^{3}},
\end{aligned}
$$

where

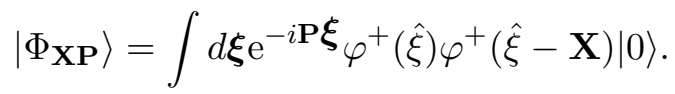

Remember that in the variables $\hat{\xi}_{1}, \hat{\xi}_{2}, \hat{\xi}_{1}^{\prime}, \hat{\xi}_{2}^{\prime}$, and $\hat{\xi}, \hat{\xi}^{\prime}$ the quantity $\xi^{0}$ has the same value. For symmetry, we retain the four-dimensional notation for the variables.

It should be noted that because of the existence of the common cyclic (vacuum) vector the identity resolution in the subspace of three-particle states cannot be in any way represented as a tensor product of the corresponding identity resolutions in the subspaces of one- and two-particle states. Unlike the non-relativistic case, the identity resolution (26) for the relativistic quantum field cannot be reduced to the form defined by Eq.(16).

The measurement (26) corresponds to the situation where the observation is only performed on the two particles of three, while the third particle is not involved in the measurement.

The probability of obtaining an outcome in the neighbourhood $d \mathbf{X} d \mathbf{P}$ of the point $\mathbf{X P}$ of the space of possible outcomes is given by the standard expression

$$
\operatorname{Pr}\{d \mathbf{X} d \mathbf{P}\}=\operatorname{Tr}\left\{|\Psi\rangle\langle\Psi| \mathcal{M}_{\mathcal{H}}(d \mathbf{X} d \mathbf{P})\right\}=
$$




$$
\left(\int d \mathbf{x}|\mathcal{A}(\mathbf{x}, \mathbf{X P})|^{2}\right) d \mathbf{X} d \mathbf{P}
$$

where the total transition amplitude $\mathcal{A}(\mathbf{x}, \mathbf{X P})$ is defined as

$$
\begin{gathered}
\mathcal{A}(\mathbf{x}, \mathbf{X P})=\iiint d \hat{x}^{\prime} d \mathbf{x}_{1} d \boldsymbol{\xi} \mathrm{e}^{i \mathbf{P} \boldsymbol{\xi}} f\left(\hat{x}^{\prime}\right) \\
\left\langle 0\left|\varphi^{-}(\hat{\xi}) \varphi^{-}(\hat{\xi}-\mathbf{X}) \varphi^{-}(\hat{x}) \varphi^{+}\left(\hat{x}_{1}\right) \varphi^{+}\left(\hat{x}_{1}\right) \varphi^{+}\left(\hat{x}^{\prime}\right)\right| 0\right\rangle,
\end{gathered}
$$

where two coordinates $\hat{x}_{1}, \hat{x}_{1}$ belong to the EPR-pair, the variables $\hat{x}$ and $\hat{x}^{\prime}$ correspond to the packet with the shape $f\left(\hat{x}^{\prime}\right)$ being teleported, and, finally, the coordinates $\hat{\xi}, \hat{\xi}-\mathbf{X}$ refer to the measurement.

It should be noted that for the relativistic quantum field there exists no analogy for the expression (2). The knowledge of measurement (an operator valued measure $\mathcal{M}(d \theta)$ ) itself is not sufficient to tell what states is the quantum system in after the measurement which gave a particular outcome. To answer this question one should know the instrument (superoperator) generating the indicated operator valued measure. However, the superoperator cannot be uniquely recovered from the given operator valued measure. Fortunately, in the non-relativistic quantum mechanics it is sufficient to know only the measurement itself to completely describe the state of the teleported particle [5]. The latter is explained by the possibility of the representation of the measurement itself (an operator valued measure) as a tensor product of the appropriate identity resolutions in the subspaces of the states of constituent subsystems.

For the relativistic quantum field it is impossible to represent the measurement as a tensor product of the form (1). Therefore, asking what is the state of the teleported system after the measurement for the quantum field is physically meaningless and one can only speak of the transition amplitude of the field as a whole from one state to another.

The vacuum average in Eq. (29) is only determined by the quantum field properties. For a free field the vacuum average is decoupled into the pairwise averages $[5,6]$ so that only six contributions to the transition amplitude $\mathcal{A}$ arise.

The quantity $|\mathcal{A}(\mathbf{x}, \mathbf{X P})|^{2}$ can be interpreted as the probability of detecting the "teleported" particle at a point $\mathbf{x}$ at time $x^{0}$ if the measurement gave an outcome in the neighbourhood $(\mathbf{X}, \mathbf{P} ; \mathbf{X P}+$ $d \mathbf{X} d \mathbf{P})$. Similarly, the quantity $\mathcal{A}(\mathbf{x}, \mathbf{X P})$ is the transition amplitude for the packet from the state with the shape $f\left(\mathbf{x}^{\prime}\right)$ at time $x^{0^{\prime}}$ to the point $\mathbf{x}$ at time $x^{0}$. One has the following expression for the amplitude:

$$
\begin{gathered}
\mathcal{A}(\mathbf{x}, \mathbf{X P})=2 \iiint d \hat{x}^{\prime} d \mathbf{x}_{1} d \boldsymbol{\xi} \mathrm{e}^{i \mathbf{P} \boldsymbol{\xi}} f\left(\hat{x}^{\prime}\right) \\
\left\{\mathcal{D}_{m}^{+}\left(\hat{x}_{1}-\hat{x}^{\prime}\right)\left[\mathcal{D}_{m}^{+}(\hat{x}-\hat{\xi}) \mathcal{D}_{m}^{+}\left(\hat{x}_{1}-\hat{\xi}+\mathbf{X}\right)+\mathcal{D}_{m}^{+}\left(\hat{x}_{1}-\hat{\xi}\right) \mathcal{D}_{m}^{+}(\hat{x}-\hat{\xi}+\mathbf{X})\right]+\right. \\
\left.2 \mathcal{D}_{m}^{+}\left(\hat{x}-\hat{x}^{\prime}\right) \mathcal{D}_{m}^{+}\left(\hat{x}_{1}-\hat{\xi}\right) \mathcal{D}_{m}^{+}\left(\hat{x}_{1}-\hat{\xi}+\mathbf{X}\right)\right\}
\end{gathered}
$$

where $\mathcal{D}_{m}^{+}(\hat{x})$ is the commutator distribution for a free field with mass $m$,

$$
\begin{gathered}
\mathcal{D}_{m}^{ \pm}(\hat{x})= \pm \frac{1}{i(2 \pi)^{3 / 2}} \int \mathrm{e}^{i \hat{p} \hat{x}} \theta\left( \pm p_{0}\right) \delta\left(\hat{p}^{2}-m^{2}\right) d \hat{p}= \\
\frac{1}{4 \pi} \varepsilon\left(x_{0}\right) \delta\left(\hat{x}^{2}\right) \mp \frac{i m}{8 \pi \sqrt{\hat{x}^{2}}} \theta\left(\hat{x}^{2}\right)\left[N_{1}\left(m \sqrt{\hat{x}^{2}}\right) \mp i \varepsilon\left(x_{0}\right) J_{1}\left(m \sqrt{\hat{x}^{2}}\right)\right] \pm \frac{i m}{4 \pi^{2} \sqrt{-\hat{x}^{2}}} \theta\left(-\hat{x}^{2}\right) K_{1}\left(m \sqrt{-\hat{x}^{2}}\right), \\
\varepsilon\left(x_{0}\right) \delta\left(\hat{x}^{2}\right) \equiv \frac{\delta\left(x_{0}-|\mathbf{x}|\right)-\delta\left(x_{0}+|\mathbf{x}|\right)}{2|\mathbf{x}|} .
\end{gathered}
$$

To within the exponential tails, the commutator function is zero beyond the light cone and has a singularity on its surface $\lambda^{2}=\left(\hat{x}-\hat{x}^{\prime}\right)^{2}=0$; outside the light cone the $\mathcal{D}^{ \pm}(\lambda)$-function decay exponentially at the Compton length as $|\lambda|^{-3 / 4} \exp (-m \sqrt{|\lambda|})[5,6]$. At a fixed point $\hat{x}$ contributing to the integral are only points $\hat{x}^{\prime}$ lying within the light cone issued from the point $\hat{x}$, which actually follows from the microcausality principle and impossibility of the faster-than-light field propagation. 
The amplitude (30) is actually a distribution which should be smeared with a test function to obtain a final result. It should also be noted that the product of any number of positive- or negativefrequency functions $\mathcal{D}^{ \pm}(\hat{x})$ (unlike the product of causal functions) is again correctly defined as a distribution from $\mathcal{J}^{*}(\hat{x})$ since in the momentum representation all these functions have their supports located in the front part of the light cone.

Since we are only interested in the relative probabilities of different processes, we shall directly employ the expression (30) for the amplitude.

Because of the common vacuum vector, it is impossible to arrange a measurement in which there are no contributions to the transition amplitude from the processes which are irrelevent to the teleportation. Formally, the fraction of all these irrelevant processes is $1 / 2$. This circumstance has a fundamental nature and cannot be circumvented by any geometrical tricks in the experiment.

The commutator function $\mathcal{D}_{m}^{+}(\hat{x}-\hat{y})$ describes the creation of a particle at point $\hat{x}$, its propagation, and destruction at point $\hat{y}$ (for $\left.y^{0}>x^{0}\right)[5,6]$

$$
\left\langle 0\left|\varphi^{-}(\hat{y}) \varphi^{+}(\hat{x})\right| 0\right\rangle=-i \mathcal{D}_{m}^{+}(\hat{x}-\hat{y}) .
$$

Further, the Lorentz-invariant scalar product

$$
\begin{gathered}
\left(\varphi^{-}(f), \varphi^{+}(g)\right)=\left\langle 0\left|\varphi^{-}(f) \varphi^{+}(g)\right| 0\right\rangle=\iint d \hat{x} d \hat{y} f^{*}(\hat{y}) \mathcal{D}_{m}^{+}(\hat{x}-\hat{y}) g(\hat{x})= \\
\iint d \hat{x} d \hat{y} f^{*}(\hat{y})\left\langle 0\left|\varphi^{-}(\hat{y}) \varphi^{+}(\hat{x})\right| 0\right\rangle g(\hat{x})
\end{gathered}
$$

is interpreted as the amplitude of the packet transition from the state with the "shape" $g(\hat{x})$ to the state with the "shape" $f(\hat{y})$. Since the test functions $g(\hat{x})$ and $f(\hat{y})$ determine the state of the field through their values on the mass shell only, it is convenient to rewrite the amplitude in the form

$$
\left(\varphi^{-}(f), \varphi^{+}(g)\right)=-\frac{i}{(2 \pi)^{3 / 2}} \int d \hat{p} \theta\left(p^{0}\right) \delta\left(\hat{p}^{2}-m^{2}\right) f^{*}(\hat{p}) g(\hat{p})
$$

where $f(\hat{p})$ and $g(\hat{p})$ are the four-dimensional Fourier transforms of the functions $f(\hat{x})$ and $g(\hat{x})$,

$$
f(\hat{p})=\frac{1}{(2 \pi)^{3 / 2}} \int d \hat{x} \mathrm{e}^{-i \hat{p} \hat{x}} f(\hat{x}) .
$$

Integration over the mass shell in Eq. (34) yields

$$
\begin{gathered}
\left(\varphi^{-}(f), \varphi^{+}(g)\right)=-\frac{i}{(2 \pi)^{3 / 2}} \int_{V_{m}^{+}} \frac{d \mathbf{p}}{2 p_{0}} f^{*}(\mathbf{p}) \mathrm{e}^{i p^{0} y^{0}} g(\mathbf{p}) \mathrm{e}^{-i p^{0} x^{0}}= \\
\iint d \mathbf{x} d \mathbf{y} f^{*}(\mathbf{y}) \mathcal{D}_{m}^{+}(\hat{x}-\hat{y}) g(\mathbf{x}),
\end{gathered}
$$

where the commutator function is defined as

$$
\mathcal{D}_{m}^{+}(\hat{x}-\hat{y})=-\frac{i}{(2 \pi)^{3 / 2}} \int_{V_{m}^{+}} \frac{d \mathbf{p}}{2 p_{0}} \mathrm{e}^{i\left[\mathbf{p}(\mathbf{x}-\mathbf{y})-i p^{0}\left(x^{0}-y^{0}\right)\right]} \quad \hat{x}=\left(x^{0}, \mathbf{x}\right), \quad \hat{y}=\left(y^{0}, \mathbf{y}\right),
$$

and

$$
f(\mathbf{x})=\frac{1}{(2 \pi)^{3 / 2}} \int d \mathbf{p} \mathrm{e}^{-i \mathbf{p x}} f(\mathbf{p}) .
$$

The values of the test functions $f(\mathbf{p}) \mathrm{e}^{i p^{0} y^{0}}$ and $g(\mathbf{p}) \mathrm{e}^{-i p^{0} x^{0}}$ on the mass shell uniquely determine the state and are interpreted as the packet shape in the momentum representation. In the position representation the quantities $f(\mathbf{x})$ and $g(\mathbf{y})$ are interpreted as the spatial shape of the packet at times $x^{0}$ and $y^{0}$. Note that the factors $\mathrm{e}^{i p^{0} y^{0}}$ and $\mathrm{e}^{-i p^{0} x^{0}}$ refer to the packet shape (one could simply write $\tilde{f}(\mathbf{p})=f(\mathbf{p}) \mathrm{e}^{i p^{0} y^{0}}$ and similarly for $\left.g(\mathbf{p})\right)$ and have nothing to do with the dummy integration variable in Eq. (33). This representation is chosen because in this form the Lorentz-invariant scalar product 
(36) has the meaning of the transition amplitude from the state which at time $x^{0}$ has the spatial shape $g(\mathbf{x})$ to the state with the spatial shape $f(\mathbf{x})$ by the time $y^{0}$. To within the exponentially decreasing tails at the Compton length outside the light cones, the contributions to this amplitude are only given by the points lying inside the light cones issued from each point $\hat{x}=\left(x^{0}, \mathbf{x}\right)\left(|\mathbf{x}-\mathbf{y}|^{2}-\left|x^{0}-y^{0}\right|^{2}<0\right)$ where the function $g(\mathbf{x})$ is different from zero.

Then in a similar way Eq.(30) can be rewritten in the form where the integration is only performed over the spatial coordinates

$$
\begin{gathered}
\mathcal{A}(\mathbf{x}, \mathbf{X P})=2 \iiint d \mathbf{x}^{\prime} d \mathbf{x}_{1} d \boldsymbol{\xi} \mathrm{e}^{i \mathbf{P} \boldsymbol{\xi}} f\left(\mathbf{x}^{\prime}\right) \\
\left\{\mathcal{D}_{m}^{+}\left(\hat{x}_{1}-\hat{x}^{\prime}\right)\left[\mathcal{D}_{m}^{+}(\hat{x}-\hat{\xi}) \mathcal{D}_{m}^{+}\left(\hat{x}_{1}-\hat{\xi}+\mathbf{X}\right)+\mathcal{D}_{m}^{+}\left(\hat{x}_{1}-\hat{\xi}\right) \mathcal{D}_{m}^{+}(\hat{x}-\hat{\xi}+\mathbf{X})\right]+\right. \\
\left.2 \mathcal{D}_{m}^{+}\left(\hat{x}-\hat{x}^{\prime}\right) \mathcal{D}_{m}^{+}\left(\hat{x}_{1}-\hat{\xi}\right) \mathcal{D}_{m}^{+}\left(\hat{x}_{1}-\hat{\xi}+\mathbf{X}\right)\right\}
\end{gathered}
$$

where the quantity $f(\mathbf{x})$ has the meaning of the spatial shape of the unknown packet to be teleported at time $x^{0}\left(\hat{x}=\left(x^{0}, \mathbf{x}\right)\right)$. The quantity $x^{0}$ appears as a parameter in the arguments of the commutator functions. The rest variables $x_{1}^{0}, x^{0^{\prime}}, \xi^{0}$ also appear in the arguments in $\hat{x}_{1}, \hat{x}_{1}^{\prime}, \hat{\xi}^{\prime}$ as parameters. This form is best suitable for interpretation.

For example, the first term in Eq. (39) yields the amplitude of the process associated with the creation of a non-local EPR-pair state (formally, instantaneously in the entire space, as indicated by the integral over $\mathbf{x}_{1}$ ) at time $x_{1}^{0}$, propagation of the packet in an unknown state which at time $x^{0}$ has the shape $f(\mathbf{x})$ and subsequent joint measurement (also non-local, the integral over $\boldsymbol{\xi}$ ) at time $\xi^{0}$ performed on the particle in the unknown state and one of the particles of the EPR-pair. In addition, one of the factors describes the free propagation of the second particle in the EPR-pair, which is not involved in the measurement, to the point $\hat{x}=\left(x^{0}, \mathbf{x}\right)$. The second term in Eq. (39) has a similar interpretation. The last two terms describe the processes irrelevant to the teleportation. They describe the contributions to the amplitudes corresponding to the processes where the measurement affects only the two particles of the EPR-pair while the particle whose state is to be teleported propagates freely.

Although the transition to the non-relativistic theory cannot be performed literately, it is still interesting to mention the formal algorithm for this transition: One should omit all the terms in the transition amplitude associated with the particle permutations and replace the commutator distributions $\mathcal{D}_{m}^{-}(\hat{x})$ by ordinary $\delta$-functions. Note that because of the singularity, this replacement can only be understood symbolically.

The replacement of $\mathcal{D}_{m}^{+}(\hat{x})$-functions by ordinary $\delta(\mathbf{x})$-functions is required because in the nonrelativistic case the integration is performed with the Galilei-invariant measure $d \mu(\mathbf{p})=d \mathbf{p}$, while in the relativistic theory the Lorentz-invariant measure $d \mu(\mathbf{p})=\theta\left(p^{0}\right) \delta\left(\hat{p}^{2}-m^{2}\right) d \hat{p}=d \mathbf{p} /\left.2 p^{0}\right|_{V_{m}^{+}}$is employed which finally gives

$$
\mathcal{D}_{m}^{+}(\hat{x})=-\frac{1}{(2 \pi)^{3 / 2}} \int_{V_{m}^{+}} \frac{d \mathbf{p}}{2 p_{0}} \mathrm{e}^{i \hat{p} \hat{x}} \rightarrow \frac{i}{(2 \pi)^{3 / 2}} \int d \mathbf{p} \mathrm{e}^{i \mathbf{p x}}=\delta(\mathbf{x}) .
$$

The temporal phase factors in the non-relativistic case do not matter because of the absence of any limitations on the propagation speed. Finally, the partial amplitude of the transition from point $\mathbf{x}^{\prime}$ at time $x^{0^{\prime}}$ to the point $\mathbf{x}$ at time $x^{0}$ we have

$$
\begin{gathered}
\mathcal{A}\left(\mathbf{x}^{\prime}, \mathbf{x}, \mathbf{X P}\right)= \\
2 f(\mathbf{x}) \mathrm{e}^{i \mathbf{P} \mathbf{x}} \delta\left(\mathbf{x}-\mathbf{x}^{\prime}+\mathbf{X}\right)+2 f(\mathbf{x}) \mathrm{e}^{i \mathbf{P}(\mathbf{x}+\mathbf{X})} \delta\left(\mathbf{x}-\mathbf{x}^{\prime}+\mathbf{X}\right)+4 f(\mathbf{x}) \delta(\mathbf{X}) \delta(\mathbf{P}), \\
\mathcal{A}(\mathbf{x}, \mathbf{X P})=\int d \mathbf{x}^{\prime} \mathcal{A}\left(\mathbf{x}^{\prime}, \mathbf{x}, \mathbf{X P}\right),
\end{gathered}
$$

where the partial amplitude for the transition from point $\mathbf{x}^{\prime}$ at time $x^{0^{\prime}}$ to the point $\mathbf{x}$ at time $x^{0}$ "weighted" with the packet shape $f\left(\mathbf{x}^{\prime}\right)$ is introduced. 
If the contribution of only the first term in Eq. (41) to $\mathcal{A}\left(\mathbf{x}^{\prime}, \mathbf{x}, \mathbf{X P}\right)$ is understood literally as the amplitude of the transition to the point $\mathbf{x}$ under the condition that the measurement gave an outcome in the interval $(\mathbf{X}, \mathbf{P} ; \mathbf{X P}+d \mathbf{X} d \mathbf{P})$, this amplitude coincides (to within an obvious unitary transformation which is only determined by the measurement outcome, i.e. the value of the pair $\mathbf{X}, \mathbf{P}$ ) with the amplitude of propagation of the wave packet having the shape $f\left(\mathbf{x}^{\prime}\right)$ at the initial moment of time $x^{0^{\prime}}$ to the final point with coordinates $\mathbf{x}, x^{0}$

$$
\mathcal{A}(\mathbf{x}, \mathbf{X P})=f\left(\mathbf{x}^{\prime}-\mathbf{X}\right) \mathrm{e}^{i \mathbf{P}\left(\mathbf{x}^{\prime}-\mathbf{X}\right)} .
$$

For the probabilities (again understood symbolically) of obtaining different measurement outcomes we have

$$
\begin{gathered}
\operatorname{Pr}\{d \mathbf{X} d \mathbf{P}\}=\left(\int d \mathbf{x}|\mathcal{A}(\mathbf{x}, \mathbf{X} \mathbf{P})|^{2}\right) \frac{d \mathbf{X} d \mathbf{P}}{(2 \pi)^{3}}= \\
\left(\int d \mathbf{x}|f(\mathbf{x})|^{2}\right) \frac{d \mathbf{X} d \mathbf{P}}{(2 \pi)^{3}}=\frac{d \mathbf{X} d \mathbf{P}}{(2 \pi)^{3}}
\end{gathered}
$$

just as the ideal teleportation requires, the probabilities of obtaining various measurement outcomes do not depend on the unknown state which is to be teleported.

The second term in the amplitude (41) also refers to the teleportation process where one of the particles of the EPR-pair and the particle in the unknown state are exchanged (compared with the teleportation process described by the first term in Eq. (41)).

The last term in Eq. (41) is irrelevant to the teleportation and arises when the measurement only affects the two particles of the EPR-pair (there are two equal contributions because of the exchange of the particles within the EPR-pair). These processes contribute only at the point $\mathbf{X}=0, \mathbf{P}=0$ of the outcome space and their effect can in principle be eliminated by simply discarding the measurements which gave this result.

Nevertheless, the first two terms in Eq. (41) describing the teleportation process have different phase factors which does not allow to correctly modify the transition amplitude by a unitary transformation similar to the non-relativistic case (this would be possible if only the first term were present).

At a first glance, one could simply keep only the measurements which gave the results with $\mathbf{P}=0$ (when the phase factors are identical). However, in that case the contribution of the "parasitic" processes when the unknown packet is not affected by the measurement and propagates freely becomes essential because of the $\delta$-functions $(\delta(\mathbf{X}) \delta(\mathbf{P}))$. Under these conditions it is impossible to distinguish between the teleportation and free propagation contributions to the transition amplitude.

In spite of the fact that the parasitic terms cannot be eliminated, their contribution is only important in the vicinity of the point $\mathbf{X}=0, \mathbf{P}=0$ and has zero measure. Although being rather strange at a first glance, this circumstance has a simple qualitative interpretation related to the fact that each pure state in the infinite-dimensional Hilbert space has in a certain sense zero measure, as it is most simply explained in the non-relativistic theory. The EPR-pair state is written as

$$
|\psi\rangle_{E P R}=\frac{1}{(2 \pi)^{3}} \int d \mathbf{k}|\mathbf{k}\rangle_{1} \otimes|\mathbf{k}+\mathbf{q}\rangle_{2}
$$

with a fixed q. The parasitic terms correspond to the measurement performed on the two particles of the EPR-pair which is actually reduced to the projection on the state

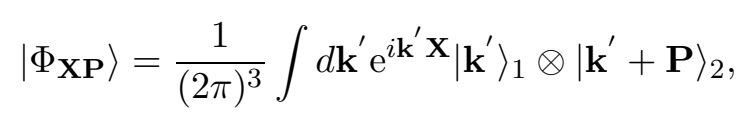

which in fact is another EPR-pair with a different total momentum (remember that we used the EPR-pair with $\mathbf{q}=0$ ). For the projection we have

$$
\left\langle\Phi_{\mathbf{X P}} \mid \psi\right\rangle_{E P R} \propto \delta(\mathbf{P}) \int d \mathbf{k} \mathrm{e}^{i \mathbf{k X}}=\delta(\mathbf{X}) \delta(\mathbf{P}),
$$

where the $\delta$-functions should be understood as indicated in the above discussion. 
The latter means that the measure of an individual EPR-pair with a fixed $\mathbf{q}$ among the entire set of all EPR-pairs is zero. Therefore, the probability of occurrence of a particular EPR-pair with a specified $\mathbf{q}$ is zero (the measurement runs over the entire set of EPR-pairs).

Thus, in the relativistic quantum field theory the existence of a common cyclic vector state together with the microcausality principle (commutation relations) make the quantum teleportation impossible in the sense it is understood in the non-relativistic quantum mechanics.

It should be emphasized once again that all the above arguments are only applicable to the teleportation of a completely unknown field state. In that case there is no way to "label" the individual particles involved in the teleportation procedure. However, if the state to be teleported is only partly unknown (e.g. for the case of photon field only the polarization state is unknown while the photon momentum and the total momentum of the EPR-pair are specified beforehand), the available information can be used to construct the "labels" distinguishing the identical particles [9].

This work was supported by the Russian Foundation for Basic Research (project No 99-02-18127), the project "Physical Principles of the Quantum Computer", and the program "Advanced Devices and Technologies in Micro- and Nanoelectronics".

This work was also supported by the Wihuri Foundation, Finland.

\section{References}

[1] A.Einstein, B.Podolsky, and N.Rosen, Phys. Rev., 47, 777 (1935).

[2] C.H.Bennett, G.Brassard, C.Crepeau, R.Jozsa, A.Peres, and W.K.Wootters, Phys. Rev. Lett., 70, 1895 (1993).

[3] L.Vaidman, Phys. Rev., A49, 1473 (1994).

[4] S.N.Molotkov and S.S.Nazin, JETP, 89, 413 (1999); /quant-ph/9906018, On the Teleportation of Continuous Variable.

[5] N.N.Bogolubov, A.A.Logunov, and I.T.Todorov, Foundations of the Axiomatic Approach to the Quantum Field Theory, Moscow, "Nauka", 1969.

[6] N.N.Bogolubov, A.A.Logunov, A.I.Oksak, and I.T.Todorov, General Principles of the Quantum Field Theory, Moscow, Nauka, 1987.

[7] D.A.Kirzhnits, Usp.Fiz.Nauk, 90, 129 (1966).

[8] I.Bialynicki-Birula, Phys. Rev. Lett., 80, 5247 (1998).

[9] to be published. 